\title{
CORROSIVIDADE CAUSADA POR SOLUÇÕES PRODUZIDAS COM CREOSOTO VEGETAL ${ }^{1}$
}

\author{
Juarez Benigno Paes ${ }^{2}$, Benedito Rocha Vital ${ }^{3}$, Ricardo Marius Della Lucia ${ }^{3}$ e Terezinha Maria Castro Della Lucia ${ }^{4}$
}

\begin{abstract}
RESUMO - Esta pesquisa teve como objetivo avaliar a corrosividade de soluções preservativas preparadas com creosoto vegetal. Por destilação do alcatrão vegetal, obteve-se o creosoto vegetal bruto, recuperado à temperatura de $110-255^{\circ} \mathrm{C}$. Uma fração deste destilado foi lavada com solução a $9 \%$ de bicarbonato de sódio, obtendo-se o creosoto vegetal purificado. Ambas as frações foram enriquecidas com 3\% de naftenato de cobre; $3 \%$ de naftenato de zinco; $3 \%$ de naftenato de cobalto; $2 \%$ de TBTO; $2 \%$ de tribromofenato de tributil-estanho; $2 \%$ de pentaclorofenol; ou $0,4 \%$ de trióxido de arsênico. Foram preparadas 16 soluções preservativas, sendo 14 enriquecidas, além do creosoto vegetal bruto e do creosoto vegetal purificado. Placas de aço SAE 1006 foram expostas por 6 horas às temperaturas de 25,45 e $100{ }^{\circ} \mathrm{C}$, à ação corrosiva dessas soluções. A corrosividade das soluções de creosoto vegetal foi comparada à corrosividade causada pelo creosoto mineral. As soluções preparadas com creosoto vegetal purificado foram menos corrosivas que suas similares preparadas com creosoto vegetal bruto, sem, no entanto, atingir a baixa corrosividade do creosoto mineral.
\end{abstract}

Palavras-chave: Creosoto vegetal, creosoto mineral e testes de corrosão.

\section{CORROSION CAUSED BY SOLUTIONS PRODUCED WITH WOOD TAR CREOSOTE}

\begin{abstract}
The objective of this research was to evaluate the corrosiveness of preservative solutions prepared with wood tar creosote. By distilling of wood tar, crude wood tar creosote was recovered at a temperature of 100 $255^{\circ} \mathrm{C}$. A fraction of this product was washed with a solution of sodium bicarbonate at $9 \%$, resulting in purified wood tar creosote. Both fractions were enriched with 3\% copper naphtenate, 3\% zinc naphtenate, 3\% cobalt naphtenate, $2 \%$ TBTO, $2 \%$ tributhyl-tin tribromophenate, $2 \%$ pentachlorophenol, or with $0.4 \%$ arsenic trioxide. A total of 16 preservative solutions were prepared of which 14 were enriched, besides the crude wood tar creosote and the purified wood tar creosote. SAE 1006 steel plates were exposed for 6 hours at temperatures of 25, 45 and $100{ }^{\circ} \mathrm{C}$, to the corrosive action of those solutions. The corrosiveness of wood tar creosote solutions was compared with that caused by coal tar creosote. The solutions with purified wood tar creosote were less corrosive than similar solutions prepared with crude wood tar creosote, although being more corrosive than the coal tar creosote.
\end{abstract}

Key words: $\quad$ Wood tar creosote, coal tar creosote and corrosion test.

\section{INTRODUÇÃO}

Nas décadas de 70 e 80 , o alcatrão vegetal obtido como subproduto da carbonização da madeira foi utilizado intensamente como insumo energético no Brasil, em substituição ao óleo combustível. Porém, passado o período da crise energética, a redução dos preços do barril de petróleo no mercado internacional inviabilizou a utilização do alcatrão vegetal para fins energéticos.

Das pesquisas para recuperação de energéticos da carbonização, desenvolveram-se tecnologias e pátios industriais para recuperação do alcatrão vegetal. No

1 Recebido para publicação em 16.11.2000.

Aceito para publicação em 6.11.2002.

2 Dep. de Engenharia Florestal da Universidade Federal da Paraíba - UFPB, 58700-970 Patos-PB, <jbp2@ uol.com.br>; ${ }^{3}$ Dep. de Engenharia Florestal da Universidade Federal de Viçosa - UFV, 36571-000 Viçosa-MG.; ${ }^{4}$ Dep. de Biologia Animal da UFV. 
entanto, hoje faltam opções para a utilização desse produto, do qual pode ser obtido o creosoto vegetal, que em virtude de seu caráter fenólico pode apresentar várias opções de utilização.

O creosoto vegetal é eficiente contra fungos e insetos xilófagos, mas poucos esforços têm sido dispensados com o intuito de destilar um produto de qualidade uniforme que pudesse se enquadrar em uma normalização técnica (Findlay, 1983).

Apesar de sua eficiência contra organismos xilófagos, os alcatrões de folhosas possuem caráter ácido. Assim sendo, seus creosotos podem ter um conteúdo ácido elevado (Hunt \& Garratt, 1967), o que não é desvantajoso, pois estes ácidos, que são principalmente o acético e o fórmico, corroem o ferro e o aço, tornando o produto inadequado para tratamentos industriais (Hunt \& Garratt, 1967; Jankowsky, 1986). Jankowsky (1986) cita que a forma mais viável de resolver esse problema é efetuar a retificação ou purificação do creosoto, visando a eliminação dos ácidos. Outra alternativa, caso o creosoto continue corrosivo, seria a adição de produtos inibidores da corrosão.

O enriquecimento das substâncias fenólicas, a purificação ou a neutralização dos ácidos do creosoto vegetal poderão trazer grandes benefícios à utilização desse preservativo, pois sendo o creosoto de madeira menos viscoso que o mineral (Richardson, 1993), ele terá grande penetração na madeira, reduzindo, assim, tempos e pressões de tratamento.

A determinação da corrosão de um produto químico pode ser em nível de laboratório ou em condições reais de serviço, em contato com a madeira tratada. Os testes de laboratório podem fornecer importantes informações sobre a interação entre líquidos preservativos e equipamentos metálicos da planta de preservação de madeiras, como também sobre o efeito da madeira tratada em metais empregados para sua fixação (Wilkinson, 1979).

A corrosão do equipamento é simulada pela submersão de placas limpas de metal em soluções preservativas. Após o período de teste, que normalmente é de seis meses, as amostras são limpas e o grau de corrosão é avaliado pela perda de massa. A metodologia adotada pela ASTM D-130 (1991) consiste na exposição de placas de cobre às temperaturas de 25,45 e $100^{\circ} \mathrm{C}$, por 6 horas.

O objetivo deste estudo foi avaliar a corrosividade de soluções preservativas preparadas com creosoto vegetal em contato com metais, em condições de laboratório.

\section{MATERIAL E MÉTODOS}

\subsection{Obtenção, Destilação e Purificação do Alcatrão Vegetal}

O alcatrão vegetal utilizado nesta pesquisa foi produzido pela Companhia Agrícola e Florestal Santa Bárbara (CAF), Bom Despacho-MG. O produto foi proveniente de fornos de alvenaria e recuperado como subproduto da carbonização das madeiras de Eucalyptus grandis e E. cloeziana ( $\pm 50 \%$ de cada espécie).

Para produção das soluções preservativas, o alcatrão foi destilado a temperaturas compreendidas entre $110 \mathrm{e}$ $225^{\circ} \mathrm{C}$, à pressão atmosférica, em retorta de cobre de 201 de capacidade, destilando-se 121 de cada vez. Os vapores da destilação foram condensados por uma bateria de três condensadores "Liebig", ligados em série.

Por meio da destilação do alcatrão, foram obtidos o alcatrão anidro e o creosoto vegetal. Já o creosoto vegetal purificado foi obtido ao lavar o creosoto vegetal bruto com uma solução de $9 \%$ de bicarbonato de sódio.

\subsection{Preparo das Soluções Preservativas}

As soluções obtidas, como descrito no item anterior, foram utilizadas puras ou enriquecidas com várias substâncias químicas, visando obter as soluções preservativas a serem utilizadas nos ensaios de corrosividade, conforme o Quadro 1.

Assim, foram obtidas 16 soluções preservativas, sendo 14 delas enriquecidas (sete com creosoto vegetal bruto e sete com creosoto vegetal purificado). As soluções foram testadas e comparadas a três testemunhas: creosoto mineral, alcatrão vegetal bruto e alcatrão vegetal anidro.

\subsection{Corrosividade das Soluções Preservativas}

Para testar a corrosividade, foram seguidas as recomendações da norma ASTM D - 130 (1991), usando placas de aço carbono SAE 1006, que é empregado na confecção das autoclaves das usinas de tratamento de madeiras. $O$ teste consiste em determinar a perda de massa de placas de metal de 12,5 x 7,5 x 1,5 cm, após 6 horas de imersão no produto a ser ensaiado, nas temperaturas de 25,45 e $100^{\circ} \mathrm{C}$. Utilizaram-se, nesta pesquisa, placas de $1,0 \mathrm{~cm}$ de espessura. 
Q uadro 1 - Soluções preservativas preparadas com creosoto vegetal Table 1 - Preservative solutions prepared with wood tar creosote

\begin{tabular}{|c|l|}
\hline Creosoto Vegetal & Tratamento/Solução Preservativa \\
\hline \multirow{3}{*}{1 - Creosoto vegetal bruto } & 1 - creosoto 1 puro \\
& 2 - creosoto $1+3 \%$ de naftenato de cobre \\
& 3 - creosoto $1+3 \%$ de naftenato de zinco \\
& 4 - creosoto $1+3 \%$ de naftenato de cobalto \\
& 5 - creosoto $1+2 \%$ de óxido de tributil-estanho (TBTO) \\
& 6 - creosoto $1+2 \%$ de tribromofenato de tributil-estanho \\
& 7 - creosoto $1+2 \%$ de pentaclorofenol \\
& 8 - creosoto $1+0,4 \%$ de trióxido de arsênico \\
\hline \multirow{3}{*}{ - Creosoto vegetal purificado } & 9 - creosoto 2 puro \\
& 10 - creosoto $2+3 \%$ de naftenato de cobre \\
& 11 - creosoto $2+3 \%$ de naftenato de zinco \\
& 12 - creosoto $2+3 \%$ de naftenato de cobalto \\
& 13 - creosoto $2+2 \%$ de óxido de tributil-estanho (TBTO) \\
& 14 - creosoto $2+2 \%$ de tribromofenato de tributil-estanho \\
& 15 - creosoto $2+2 \%$ de pentaclorofenol \\
& 16 - creosoto $2+0,4 \%$ de trióxido de arsênico \\
\hline Testemunhas & 17 - creosoto mineral \\
& 18 - alcatrão vegetal bruto \\
& 19 - alcatrão vegetal anidro \\
\hline
\end{tabular}

\subsection{Avaliação dos Resultados}

Para avaliar os resultados da corrosão causada às placas de metal, foi empregado o delineamento inteiramente casualizado, com arranjo fatorial.

$\mathrm{Na}$ comparação da corrosividade das soluções de creosoto vegetal com as testemunhas, analisaram-se os seguintes fatores: solução, com 19 níveis: temperatura, com três níveis; e a interação entre estes fatores.

$\mathrm{Na}$ análise do efeito da purificação do creosoto vegetal em suas características corrosivas, os tratamentos (Quadro 1), foram agrupados, em função dos produtos químicos utilizados para enriquecimento, e analisados sem a presença das testemunhas. Neste caso, analisaramse os seguintes fatores: preservativo, com oito níveis; creosoto, com dois níveis; temperatura, com 3 níveis; e a interação entre os fatores. $\mathrm{O}$ resultado desta análise é apresentado e discutido logo após as análises completas, em que todos os tratamentos foram analisados. Para permitir as análises, os dados de perda de massa $\left(\mathrm{mg} / \mathrm{cm}^{2}\right)$ das placas de metal foram transformados em raiz (perda de massa $+0,5$ ). Na análise e avaliação dos ensaios foi empregado o teste de Tukey, em nível de 5\% de probabilidade.

\section{RESULTADOS E DISCUSSÃO}

Observou-se que a corrosividade do creosoto vegetal, purificado ou não, foi superior àquela do creosoto mineral. As maiores corrosividades foram observadas para as soluções enriquecidas com naftenato de cobre. Constatou-se, também, que a corrosividade das soluções aumentou com o acréscimo na temperatura, o que poderá inviabilizar o uso do creosoto vegetal bruto em instalações industriais.

É citado por alguns autores, dentre eles Hartford (1973) e Wilkinson (1979), que o creosoto mineral enriquecido com pentaclorofenol causa problemas de corrosão na usina de tratamento. Porém, para o creosoto vegetal, esse enriquecimento causou decréscimo na corrosividade do produto.

R. Árvore, Viçosa-MG, v.26, n.5, p.621-627, 2002 
A remoção dos ácidos carboxílicos causou decréscimo na corrosividade, com destaque para as soluções 11, 12 e 13, que apresentaram os menores poderes corrosivos, mesmo à temperatura de $100^{\circ} \mathrm{C}$, sem, no entanto, atingir os índices do creosoto mineral.

Fato interessante é que o alcatrão anidro foi menos corrosivo que o creosoto vegetal, o que se deve, talvez, à polimerização de seus componentes, principalmente dos grupos $\mathrm{OH}$, que estão livres no creosoto vegetal, causando assim maior efeito corrosivo.

A análise de variância indicou que houve diferenças significativas na corrosividade das soluções. Essa corrosividade foi afetada de forma diferenciada, entre as várias soluções, pela temperatura.

Pelo desdobramento dos efeitos e pela análise das médias (Quadro 2), observa-se, para chapas expostas à ação corrosiva da solução preservativa a $25^{\circ} \mathrm{C}$, que o tratamento 2 (creosoto vegetal bruto enriquecido com naftenato de cobre) foi a solução mais corrosiva, porém dela não diferiram os tratamentos $1,5,4,6,7$ e 14 . O tratamento 8 não diferiu do grupo anterior, porém diferiu do tratamento 2 .

Os tratamentos 4, 6, 7, 14, 8, 15, 13, 3, 16, 9, 10, 11 e 18 não diferiram estatisticamente entre si. $\mathrm{O}$ tratamento 12 diferiu do 4, porém não diferiu dos demais. O tratamento 15 diferiu do tratamento 1 . Já os tratamentos 19 e 17 não diferiram entre si, sendo superiores aos demais. Estes foram os tratamentos que causaram menor perda de massa em virtude da corrosão, para a temperatura analisada.

Para a exposição a $45^{\circ} \mathrm{C}$, o tratamento 2 apresentou corrosividade superior à dos demais. Os tratamentos $1 \mathrm{e}$ 7 não diferiram entre si. Os tratamentos 7, 18 e 10 também foram semelhantes.

As chapas de metal submetidas aos tratamentos 18, 10, 6, 8, 4 e 5 apresentaram perdas semelhantes, em virtude do efeito da corrosividade. $\mathrm{O}$ efeito causado pelas soluções $6,8,4,5,9,15,14,13,12,3,16$ e 11 foi semelhante.

A exemplo da exposição a $25^{\circ} \mathrm{C}$, o creosoto vegetal anidro e o creosoto mineral (soluções 17 e 19, respectivamente) não diferiram estatisticamente entre si, sendo os menos corrosivos.

Para a exposição a $100{ }^{\circ} \mathrm{C}$, constatou-se, mais uma vez, superioridade do efeito corrosivo causado pelo enriquecimento do creosoto vegetal bruto com naftenato de cobre. Esse efeito corrosivo foi seguido pelo alcatrão bruto e pelas soluções $1,10,7$ e 4 , tendo os tratamentos 18 e 1 diferido entre si. Os tratamentos 5, 6, 3 e 13 foram semelhantes, diferindo dos demais tratamentos. Os tratamentos 9 e 12; e 15, 14 e 18 foram iguais.

Os resultados das soluções 8, 11 e 16 foram semelhantes aos do alcatrão anidro, sendo estas as soluções derivadas do creosoto vegetal que causaram menor corrosividade. O tratamento 17 (creosoto mineral) foi a solução que menos efeito corrosivo causou às chapas de metal.

Analisando os efeitos das temperaturas na corrosão causada pelas soluções preservativas, nota-se que a exposição a $100{ }^{\circ} \mathrm{C}$ foi a que causou maiores perdas de massa, em virtude da corrosividade, seguida das exposições a 45 e a $25^{\circ} \mathrm{C}$. Esse comportamento foi similar para as soluções 1, 2, 6, 7, 8, 9, 10, 12, 13 e 15, que foram semelhantes ao alcatrão bruto e anidro. Tal fato indica que tais soluções, em termos de corrosividade, foram bastante influenciadas pelo efeito da temperatura, não sendo, assim, indicadas para tratamento da madeira em instalações industriais, pois o aumento da corrosividade com a temperatura do tratamento poderia por em risco os equipamentos da planta de preservação de madeiras, bem como a integridade física dos operários.

Para as soluções 3, 4, 5, 11, 14 e 16, observou-se que o efeito corrosivo não foi tão pronunciado, pois as temperaturas de 25 e $45{ }^{\circ} \mathrm{C}$ causaram comportamento semelhante, indicando que tais soluções se mantiveram estáveis à corrosividade. Porém, os resultados não são comparáveis ao resultado apresentado pelo creosoto mineral, que se manteve constante para as três temperaturas estudadas, além de ser a solução que causou menor corrosividade nas placas de metal.

$\mathrm{Na}$ análise de variância em que se analisou o efeito da purificação do creosoto vegetal em suas características corrosivas, observou-se que todos os efeitos analisados acusaram ser significativos. Analisando, em separado, as médias do efeito da purificação do creosoto em cada temperatura (Quadro 3), nota-se que as soluções produzidas com creosoto vegetal bruto foram mais corrosivas, para as três temperaturas a que foram submetidas as placas de aço SAE 1006.

Analisando o efeito da temperatura de ensaio nos creosotos, observou-se que a $100{ }^{\circ} \mathrm{C}$ obteve-se a situação mais corrosiva para ambos os creosotos (purificado ou não). À temperatura de $25^{\circ} \mathrm{C}$ observou-se a situação menos corrosiva, e a $45^{\circ} \mathrm{C}$ observou-se efeito intermediário entre ambas. 
Corrosividade Causada por Soluções com Creosoto Vegetal ...

Quadro 2 - Comparações entre médias, pelo teste de Tukey, para a perda de massa ( $\left.\mathrm{mg} / \mathrm{cm}^{2}\right)$, para todas as soluções, nas temperaturas de 25,45 e $100{ }^{\circ} \mathrm{C}$

Table 2 - Comparison among averages, by the Tukey's test for mass loss (mg/ $\mathrm{cm}^{2}$ ) for all solutions at 25,45 and $100{ }^{\circ} \mathrm{C}$

\begin{tabular}{|c|c|c|c|c|c|c|c|c|}
\hline \multicolumn{9}{|c|}{ Efeito da Ação Corrosiva das Soluções em Cada Temperatura de Ensaio } \\
\hline \multicolumn{3}{|c|}{$25^{\circ} \mathrm{C}$} & \multicolumn{3}{|c|}{$45^{\circ} \mathrm{C}$} & \multicolumn{3}{|c|}{$100^{\circ} \mathrm{C}$} \\
\hline Sol. & Méd. Verd. & Comp. & Sol. & Méd. Verd. & Comp. & Sol. & Méd. Verd. & Comp. \\
\hline 2 & 0,944 & $\bar{a}$ & 2 & 2,299 & $\bar{a}$ & 2 & 7,969 & $\bar{a}$ \\
\hline 1 & 0,909 & $a b$ & 1 & 1,724 & b & 18 & 7,068 & b \\
\hline 5 & 0,901 & $a b c$ & 7 & 1,578 & $\mathrm{bc}$ & 1 & 5,816 & $\mathrm{c}$ \\
\hline 4 & 0,798 & abcd & 18 & 1,269 & $\mathrm{~cd}$ & 10 & 4,675 & d \\
\hline 6 & 0,738 & abcde & 10 & 1,235 & cde & 7 & 4,589 & d \\
\hline 7 & 0,729 & abcde & 6 & 1,038 & def & 4 & 4,572 & $\mathrm{~d}$ \\
\hline 14 & 0,643 & abcde & 8 & 0,935 & def & 5 & 3,371 & e \\
\hline 8 & 0,626 & bcde & 4 & 0,918 & def & 6 & 3,277 & e \\
\hline 15 & 0,592 & cde & 5 & 0,918 & def & 3 & 3,234 & e \\
\hline 13 & 0,583 & de & 9 & 0,884 & ef & 13 & 3,088 & e \\
\hline 3 & 0,583 & $\mathrm{de}$ & 15 & 0,867 & f & 9 & 2,565 & $\mathrm{f}$ \\
\hline 16 & 0,558 & de & 14 & 0,841 & $\mathrm{f}$ & 12 & 2,368 & $\mathrm{f}$ \\
\hline 9 & 0,549 & de & 13 & 0,832 & $\mathrm{f}$ & 15 & 1,836 & g \\
\hline 10 & 0,549 & de & 12 & 0,755 & $\mathrm{f}$ & 14 & 1,767 & $\mathrm{~g}$ \\
\hline 11 & 0,523 & de & 3 & 0,755 & $\mathrm{f}$ & 8 & 1,518 & $\mathrm{~g} \mathrm{~h}$ \\
\hline 18 & 0,515 & de & 16 & 0,729 & $\mathrm{f}$ & 11 & 1,270 & $\mathrm{~h}$ \\
\hline 12 & 0,480 & $\mathrm{e}$ & 11 & 0,712 & f & 16 & 1,244 & h \\
\hline 19 & 0,052 & $\mathrm{f}$ & 19 & 0,052 & $\mathrm{~g}$ & 19 & 1,192 & $\mathrm{~h}$ \\
\hline 17 & 0,000 & $\mathrm{f}$ & 17 & 0,026 & $\begin{array}{l}0 \\
\mathrm{~g}\end{array}$ & 17 & 0,017 & $\mathrm{i}$ \\
\hline \multicolumn{9}{|c|}{ Efeito das Temperaturas na Corrosão Causada pelas Soluções Preservativas } \\
\hline $\begin{array}{c}\text { Temp. } \\
\left({ }^{\mathrm{O}} \mathrm{C}\right)\end{array}$ & Méd. Verd. & Comp. & $\begin{array}{c}\text { Temp. } \\
\left({ }^{\mathrm{O}} \mathrm{C}\right)\end{array}$ & Méd. Verd. & Comp. & $\begin{array}{c}\text { Temp. } \\
\left({ }^{\mathrm{O}} \mathrm{C}\right)\end{array}$ & Méd. Verd. & Comp. \\
\hline \multicolumn{3}{|c|}{ Solução 1} & \multicolumn{3}{|c|}{ Solução 2} & \multicolumn{3}{|c|}{ Solução 3} \\
\hline 100 & 5,816 & $\mathrm{a}$ & 100 & 7,969 & a & 100 & 3,234 & \\
\hline 45 & 1,724 & $\mathrm{~b}$ & 45 & 2,299 & $\mathrm{~b}$ & 45 & 0,755 & $\mathrm{~b}$ \\
\hline 25 & 0,909 & $\mathrm{c}$ & 25 & 0,944 & $\mathrm{c}$ & 25 & 0,583 & b \\
\hline \multicolumn{3}{|c|}{ Solução 4} & \multicolumn{3}{|c|}{ Solução 5} & \multicolumn{3}{|c|}{ Solução 6} \\
\hline 100 & 4,572 & $\bar{a}$ & 100 & 3,371 & $\bar{a}$ & 100 & 3,277 & \\
\hline 45 & 0,918 & b & 45 & 0,918 & $\mathrm{~b}$ & 45 & 1,038 & b \\
\hline 25 & 0,798 & b & 25 & 0,901 & b & 25 & 0,738 & c \\
\hline \multicolumn{3}{|c|}{ Solução 7} & \multicolumn{3}{|c|}{ Solução 8} & \multicolumn{3}{|c|}{ Solução 9} \\
\hline 100 & 4,589 & $\mathrm{a}$ & 100 & 1,518 & $\mathrm{a}$ & 100 & $2,565^{3}$ & $\mathrm{a}$ \\
\hline 45 & 1,578 & b & 45 & 0,935 & $\mathrm{~b}$ & 45 & 0,884 & $\mathrm{~b}$ \\
\hline 25 & 0,729 & $\mathrm{c}$ & 25 & 0,626 & $\mathrm{c}$ & 25 & 0,549 & $\mathrm{c}$ \\
\hline \multicolumn{3}{|c|}{ Solução 10} & \multicolumn{3}{|c|}{ Solução 11} & \multicolumn{3}{|c|}{ Solução 12} \\
\hline 100 & 4,675 & $\mathrm{a}$ & 100 & 1,270 & $\mathrm{a}$ & 100 & 2,368 & \\
\hline 45 & 1,235 & $\mathrm{~b}$ & 45 & 0,712 & $\mathrm{~b}$ & 45 & 0,755 & $\mathrm{~b}$ \\
\hline 25 & 0,549 & $\mathrm{c}$ & 25 & 0,523 & $\mathrm{~b}$ & 25 & 0,480 & $\mathrm{c}$ \\
\hline \multicolumn{3}{|c|}{ Solução 13} & \multicolumn{3}{|c|}{ Solução 14} & \multicolumn{3}{|c|}{ Solução 15} \\
\hline 100 & 3,088 & $a$ & 100 & 1,767 & $\mathrm{a}$ & 100 & 1,836 & \\
\hline 45 & 0,832 & $\mathrm{~b}$ & 45 & 0,841 & $\mathrm{~b}$ & 45 & 0,867 & $\mathrm{~b}$ \\
\hline 25 & 0,583 & $\mathrm{c}$ & 25 & 0,643 & $\mathrm{~b}$ & 25 & 0,592 & $\mathrm{c}$ \\
\hline & Solução 16 & & & Solução 17 & & & Solução 1 & \\
\hline 100 & 1,244 & $\mathrm{a}$ & 45 & 0,026 & $\mathrm{a}$ & 100 & 7,068 & $\mathrm{a}$ \\
\hline 45 & 0,729 & $b$ & 100 & 0,017 & $\mathrm{a}$ & 45 & 1,269 & $\mathrm{~b}$ \\
\hline 25 & 0,558 & $\mathrm{c}$ & 25 & 0,000 & $\mathrm{a}$ & 25 & 0,515 & $\mathrm{c}$ \\
\hline & & & & Solução 19 & & & & \\
\hline & & & $\begin{array}{r}100 \\
45 \\
25\end{array}$ & $\begin{array}{l}1,192 \\
0,231 \\
0,052\end{array}$ & ${ }^{\mathrm{a}}{ }_{\mathrm{b}}$ & & & \\
\hline
\end{tabular}

As médias seguidas pela mesma letra não diferem entre si, a $5 \%$ de probabilidade. 
Quadro 3 - Comparações entre médias, pelo teste de Tukey, para a perda de massa (mg/cm²), considerando o efeito da purificação do creosoto vegetal nas temperaturas de 25,45 e $100{ }^{\circ} \mathrm{C}$

Table 3 - Comparison among averages, by the Tukey's test, for mass loss (mg/ cm 2), considering the purification effect of wood tar creosote at 25,45 and $100^{\circ} \mathrm{C}$

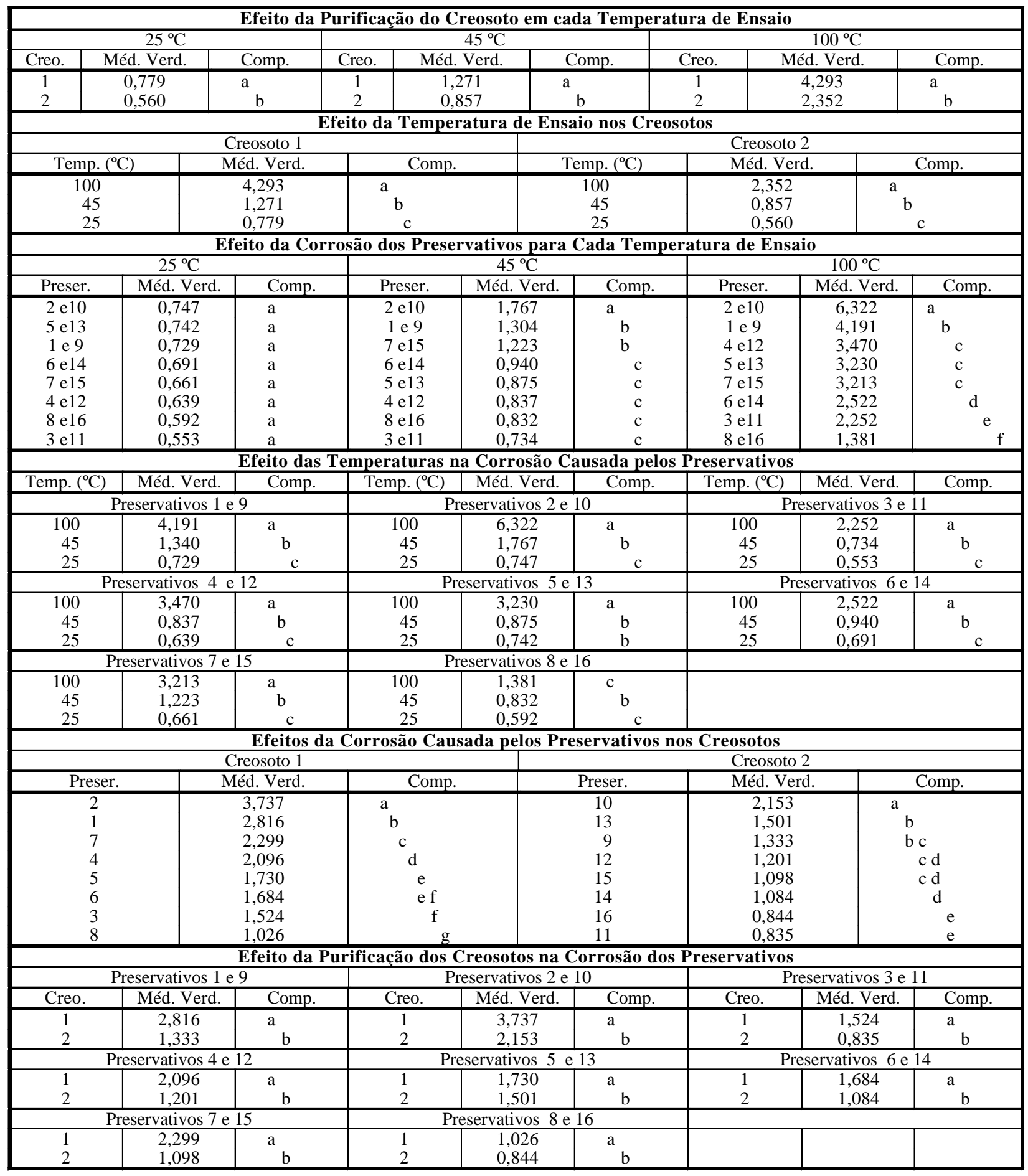

As médias seguidas pela mesma letra não diferem entre si, a $5 \%$ de probabilidade. 
Pela análise do efeito corrosivo dos preservativos para cada temperatura de ensaio, observou-se que, para $25^{\circ} \mathrm{C}$, todas as soluções preservativas causaram efeitos semelhantes. À temperatura de $45^{\circ} \mathrm{C}$, os preservativos 2 e 10 foram os mais corrosivos, seguidos pelos preservativos 1 e 9; e 7 e 15, que ocuparam valores intermediários. Os preservativos 3 e 11 foram os menos corrosivos, destes não diferindo os preservativos 6 e 14; 5 e 13; 4 e 12; e 8 e 16. À temperatura de $100^{\circ} \mathrm{C}$, os preservativos 2 e 10 foram os mais corrosivos. Seguiram-se a estes os efeitos corrosivos dos preservativos 1 e 9; 4 e 12; 5 e 13; e 7 e 15; tendo o 1 e o 9 sido os mais corrosivos entre este grupo, diferindo estatisticamente dos demais. Os preservativos 6 e 14; 3 e 11; e 8 e 16 diferiram entre si, sendo menos corrosivos que o grupo anterior. Neste caso particular, os preservativos 8 e 16 foram os menos corrosivos.

Analisando o efeito da temperatura na corrosão causada pelos preservativos, observou-se que o poder corrosivo das soluções aumentou com o incremento da temperatura. Tal fato foi observado para todos os preservativos, exceto para o creosoto vegetal enriquecido com TBTO (preservativos 5 e 13), em que o aumento na temperatura de 25 para $45^{\circ} \mathrm{C}$ não ocasionou aumento significativo na corrosão.

Para o efeito da corrosão causada pelos preservativos nos creosotos, observou-se que para as soluções produzidas com creosoto vegetal bruto (creosoto 1), o preservativo 2 (naftenato de cobre) foi o mais corrosivo e o 8 (trióxido de arsênico), o menos corrosivo. Os preservativos 3, 6 e 5 ocuparam posições intermediárias. Os produtos preservativos 1, 7 e 4 diferiram entre si; este grupo foi mais corrosivo que o anterior.

Para o creosoto purificado (creosoto 2), a solução preservativa 10 foi a mais corrosiva. As soluções 11 e 16 foram menos corrosivas, seguidas pela solução 14 (tribromofenato de tributil-estanho - "Osmose FT-40”), não tendo o seu efeito corrosivo sido diferente daquele causado pelas soluções 12 e 15 (respectivamente, naftenato de cobalto e pentaclorofenol). A solução 9 não diferiu das soluções 12 e 15 . A solução 13 (TBTO) não diferiu da solução 9, diferindo das demais dentro do mesmo grupo.

Analisando o efeito da purificação dos creosotos na corrosão dos preservativos, tem-se que as soluções provenientes do creosoto purificado foram as menos corrosivas.

\section{CONCLUSÕES}

As soluções preparadas com o creosoto vegetal são corrosivas a metais. Essa corrosividade foi reduzida pela lavagem com solução a 9\% de bicarbonato de sódio, sem, no entanto, atingir os valores apresentados pelo creosoto mineral.

A corrosividade das soluções preparadas com creosoto vegetal aumentou com a temperatura de teste, o mesmo não ocorrendo com o creosoto mineral.

O fato de as soluções preparadas com creosoto vegetal purificado serem menos corrosivas que as suas similares indicou que, com tratamentos adequados, pode-se obter produtos derivados do creosoto vegetal com boas características, para serem utilizados no tratamento da madeira.

Apesar da baixa corrosividade apresentada pelo alcatrão anidro, este não deve ser utilizado para o tratamento da madeira em instalações industriais, pois o produto apresenta alta viscosidade, o que inviabiliza as operações na indústria.

\section{REFERÊNCIAS BIBLIOGRÁFICAS}

\section{AMERICAN SOCIETY FOR TESTING AND}

MATERIALS. Standard test method for detection of copper corrosion from petroleum products by the copper strip tarnish test. Annual Book of ASTM Standards, v. 501, p. 79-84, 1991. (ASTM D-130).

FINDLAY, W. P. K. Preservative substances. In: FINDLAY, W. P. K. (Ed.). Preservation of timber in the tropics. Dordrecht: Martinus Nijhoff/Dr. W. Junk Publishers, 1985. p. 59-74.

HARTFORD, W. H. Chemical and physical properties of wood preservatives and wood-preservative systems. In: NICHOLAS, D. D. (Ed.). Wood deterioration and this preservation by preservative treatments: wood preservatives and preservative systems. Syracuse: Syracuse University Press, 1973. v. 2. p. 1-120.

HUNT, G. M.; GARRATT, G. A. Wood preservation. 3.ed. New York: Mc Graw Hill, 1967. 433 p.

JANKOWSKY, I. P. Potencialidade do creosoto de Eucalyptus spp., como preservativos para madeiras. São Paulo: USP/EP, 1986. 159 p. Tese (Doutorado em Engenharia) - Universidade de São Paulo, Escola Politécnica, 1986.

RICHARDSON, B. A. Wood preservation. 2.ed. London: E \& FN SPON, 1993. 226 p.

WILKINSON, J. G. Industrial timber preservation. London: The Rentokil Library/Associated Business-Press, 1979. $532 \mathrm{p}$.

R. Árvore, Viçosa-MG, v.26, n.5, p.621-627, 2002 